Hasking, Conducting research on nonsuicidal self-injury in schools: Ethical considerations and recommendations, 'School Psychology International' (40, 3) pp. 217-234. Copyright @ 2019. DOI: 10.1177/0143034319827056.

\title{
Conducting research on nonsuicidal self-injury in schools: Ethical considerations and
}

\section{recommendations}

\begin{abstract}
Research on nonsuicidal self-injury (NSSI) has grown significantly over the last 15 years, with much of this work focused on factors that initiate and maintain NSSI among school-aged youth. Although this work is important, it does raise several ethical concerns. In this article we outline key ethical issues underlying NSSI research in schools and offer recommendations for conducting ethically sound and productive research in this area. Ethical concerns addressed include: 1) recruitment of minors to research; 2) disclosure and confidentiality; 3) the risk of iatrogenic effects; 4) duty of care; 5) engaging schools in research; and 6) safety of the researchers. In each area, we offer recommendations to assist researchers, ethics committees, and schools in working together to conduct ethical NSSI research, further our understanding of NSSI, and address and respond to these behaviors in schools.
\end{abstract}

\section{Keywords}

ethics, nonsuicidal self-injury, NSSI, schools

Non-suicidal self-injury (NSSI), the intentional damage to one's own body tissue, without conscious suicidal intent, and for purposes not socially sanctioned (International Society for the Study of Self-Injury, 2018), is a behaviour that has increasingly captured researchers' attention. Given that NSSI typically emerges in early-mid adolescence and is most prevalent among youth (e.g., Swannell, Martin, Page, Hasking, \& St John, 2014), much of this research takes place in secondary schools. While this work is important, it comes with some inherent ethical challenges. The purpose of this paper is to draw on our collective research and clinical experience to outline key ethical issues surrounding NSSI research in school set- tings and to 
offer research-informed recommendations for addressing these issues.

\section{What is NSSI?}

Self-injury can take many forms and serve a number of functions for an individual. Common methods include cutting, scratching or burning the skin; many people who self-injure use a number of different methods, and the most common methods can vary cross-culturally (Gholamrezaei, de Stefano, \& Heath, 2017). Approximately one in five school-based adolescents report a history of NSSI (Swannell et al., 2014). Associated with psychological distress and emotional disorders (Bentley et al., 2015), NSSI is also a salient predictor of subsequent suicidal thoughts and behaviour (Ribeiro et al., 2016), which raises ethical concerns about how to safely conduct NSSI research.

\section{Ethical principles}

Since the Nuremberg Code in 1949, and the subsequent 1964 Declaration of Helsinki, moral and ethical decision making has underpinned the conduct of scientific research. Despite some variation internationally, major national and international ethical guidelines converge on three fundamental ethical principles: 1) Respect for persons; 2) Beneficence, and 3) Justice (e.g., Belmont Report, 1979; Canadian Institutes of Health Research, Natural Sciences and Engineering Research Council of Canada, and Social Sciences and Humanities Research Council of Canada 2014; Council for International Organisations of Medical Sciences, 2002; National Ethics Advisory Committee, 2012; National Health and Medical Research Council, 2007; UK Framework for Health and Social Care Research, 2018; US Title 45 Code of Federal Regulations, Part 46 (Common Rule), 2009). In addition, some guidelines explicitly value research merit and integrity, arguing that unless the research has merit and the researchers act with integrity, the research cannot be ethically justified. These principles provide a guiding 
Hasking, Conducting research on nonsuicidal self-injury in schools: Ethical considerations and recommendations, 'School Psychology International' (40, 3) pp. 217-234. Copyright @ 2019. DOI: 10.1177/0143034319827056.

framework within which ethical decisions can be made.

Respect for persons includes recognizing the autonomy of individuals to make their own decisions as well as protecting those with diminished capacity. This is most often operationalized as the need for fully informed consent to participate in research, or guardian consent where diminished capacity exists. Beneficence reflects the need for a balanced, and evidence-based, risk-benefit analysis. Ethical research is not research devoid of risk; the moral imperative is to ensure that the potential benefits (including benefits to broader society) of the research outweigh the risks. Finally, justice is ensured when all participants are treated fairly (procedural justice) and when the benefits and burdens of the research are distributed fairly (distributive justice).

Notably, these principles are also reflected in the International School Psychology Association's Code of Ethics (Oakland, Goldman, \& Bischoff, 1997), which requires school psychologists conducting research to: 1) ensure the research has benefits; 2) respect the rights of parents and students to decide whether or not to participate in research; 3) ensure students participating in research do not suffer any mental or physical distress; and 4) communicate results to educators, parents, and students. To ensure researchers follow these principles, most countries require that research be reviewed by an appropriately qualified Ethical Review Board. Some research, led by school staff, might only require Board of Education approval; however, we suggest it is best practice to also secure approval from an Ethics Review Board trained in assessing risks and benefits of research projects. Where there is no ethics review board available (e.g., remote areas), it is still important that basic ethical guidelines be consulted and followed.

\section{What ethical issues does NSSI raise in schools?}

Researchers, ethics committees, and schools often struggle with how best to con- duct school-based NSSI research. Fundamentally, the core issues concern: 1) recruiting minors to 
research; 2) disclosure and confidentiality; 3) the risk of iatrogenic effects; 4) duty of care; 5) engaging schools in research; and 6) safety of the researchers. In the remainder of this paper we outline each of these issues and offer recommendations that align with the core ethical principles underlying human research (Table 1).

\section{Recruiting minors to research}

The recruitment of students requires one to recognize the potential vulnerabilities of young people in the context of the research while also acknowledging that they are autonomous individuals with a capacity for human agency. Their decision about whether to participate in research must be respected, while the most vulnerable must be protected from coercion or exploitation. In all research projects, participants should be fully informed of the project aims, participation requirements, risks and benefits, right to withdraw, protection of privacy, procedures for accessing study results, and who to contact with any questions. In most cases, ethics committees would advocate parental consent be obtained for the participation of minors, with either assent or active consent obtained from young people. Although an opt-out approach might be appropriate with some school-based research, we would argue that, given the sensitive nature of NSSI, an opt-in approach (in which parents and students actively consent to participate in each specific project) is more appropriate.

However, NSSI is a highly secretive behaviour, often hidden from parents. If researchers are specifically interested in understanding the experience of young people who selfinjure, obtaining parental consent could put students in an awkward position in which they must disclose their self-injury to parents. Disclosure of NSSI can be a positive step, facilitating helpseeking (Hasking, Rees, Martin, \& Quigley, 2015; Kelada, Whitlock, Hasking, \& Melvin, 2016). However, disclosure, especially if it occurs as a product of the research, can impact the parent- 
Hasking, Conducting research on nonsuicidal self-injury in schools: Ethical considerations and recommendations, 'School Psychology International' (40, 3) pp. 217-234. Copyright @ 2019. DOI: 10.1177/0143034319827056.

child relationship and the family dynamic as a whole, particularly if the parents/guardians are unprepared to address NSSI with their child (Arbuthnott \& Lewis, 2015; Baetens, Andrews, Claes, \& Martin, 2015). Consequently, disclosure to parents could, in some circumstances, do more harm than good. This effect has been noted in other lines of research when seeking active parental consent (e.g. substance abuse; Kelly \& Halford, 2007).

Seeking active parental consent is also associated with lower response rates and biased samples, with parents who are less engaged or who have concerns about their child's welfare declining to give consent (Kelly \& Halford, 2007).

Further complicating decisions about whether parental consent is required is the fact that the legal of age of consent varies across (and even within) countries (from 14- to 18-years-old; European Union Agency for Fundamental Rights, 2014; Hesson, Bakal, \& Dobson, 1993; National Ethics Advisory Committee, 2012; United Nations Convention on the Rights of the Child, 1990). When possible, the child's maturity, intelligence, ability to independently make decisions (rejecting external influences), and ability to weigh risks and benefit should be considered in deciding who provides informed consent (Larcher \& Hutchinson, 2009). Regardless of age, minors and people who self-injure are usually considered vulnerable groups in the context of NSSI research. The concept of vulnerability is inherently vague, and definitions are rarely provided in ethical guidelines (Bracken-Roche, Bell, Macdonald, \& Racine, 2017). As a result, some ethical frameworks have moved away from the concept of vulnerable groups (e.g., Canadian Institutes of Health Research, Natural Sciences and Engineering Research Council of Canada, and Social Sciences and Humanities Research Council of Canada 2014). Rather than expressing vulnerability, adolescents parti- cipating in psychiatric research often report that the experience provides greater insight into themselves (Jorm et al., 2007), a notion that is mirrored 
by adolescents participating in NSSI research (Hasking, Tatnell, \& Martin, 2015).

\section{How do we balance respect, autonomy and vulnerability?}

To avoid sample bias, we recommend that, within school settings, researchers use a broader recruitment approach beyond solely seeking youth who engage in NSSI. For example, conducting a study on a range of healthy and unhealthy adolescent behaviours, of which NSSI is one, would avoid the inadvertent identification of students who self-injure. Further, we recommend that researchers be explicit about the kinds of questions that will be asked of participants. With advance knowledge of the sensitivity of the questions, participants are able to decide for themselves whether they have the maturity to reflect on their NSSI and respond in a way that will not provoke distress.

If online studies are used, researchers can consider implementing a series of brief multiple-choice questions at the end of the consent form that ask about the content of the form. This may work toward ensuring that individuals both read and understand the nature of the document. In the event that questions are answered incorrectly (e.g., after one or two attempts), participants can be directed to a page that thanks them for their interest in the study and provides appropriate resources. In keeping with efforts to ensure students fully understand what is being asked of them, it is important participants clearly understand the data management proced- ures. For example, data may be collected in an identifiable form (e.g., in interviews), but then coded (i.e., re-identifiable) for analysis, and de-identified when stored. Clear delineation of anonymity (i.e., no identifying information ever collected), and confidentiality (i.e., identifying information collected, but never shared with others) is also advised, as some youth may conflate these concepts.

Fundamental to the Declaration of Helsinki (World, Medical Association, 1964) is the 
Hasking, Conducting research on nonsuicidal self-injury in schools: Ethical considerations and recommendations, 'School Psychology International' (40, 3) pp. 217-234. Copyright @ 2019. DOI: 10.1177/0143034319827056.

right to withdraw from research. However, exactly how this right is afforded becomes less clear as research methods and technologies advance. A clear distinc- tion must be made between withdrawal of participation and withdrawal of data. For example, if participants complete an anonymous web-based survey, they are unable to withdraw their data after submitting responses. If participants elect to cease their participation (e.g., by closing the web browser), they have a right to know whether data already provided will be used in analysis. Similarly, in quali- tative studies, participants must be informed if verbatim quotes are to be used in publications. This will ensure participants understand the ramifications of with- drawing at various stages of the project. Such information must be clearly outlined in all relevant consent forms and procedures used by researchers.

\section{Disclosure and confidentiality}

Researchers should be aware that participation may itself act as disclosure, and during their participation students may unintentionally disclose sensitive information about themselves to their peers or school staff. Disclosure may also occur as an unintended result of risk management processes. That is, students may become aware that peers sought out by mental health staff have been identified as at- risk. Finally, students often participate in classrooms groups, where it is possible to see other students' responses. Given the secrecy surrounding NSSI, students are understandably concerned about the confidentiality of the information they pro- vide. Yet, teachers, school mental health staff, and some researchers have ethical and legal obligations to break confidentiality in the event that youth pose a signifi- cant risk to themselves or to others. Breaking confidentiality needs to be handled with extreme care in all instances and is one of the greatest challenges to NSSI research with minors.

\section{How do we ensure confidentiality is ethically managed?}


Limiting opportunities for unintentional disclosure could involve presenting surveys online, or in spaces where desks are separated. Limits to confidentiality must be clearly articulated. A clear protocol for breaking confidentiality should be agreed on in advance, in consultation with ethics committees and the participating schools. Researchers may encounter school administrators who feel that a single instance of NSSI constitutes 'significant risk'. Given the prevalence of NSSI in the schools, breaking confidentiality for every student with any history of NSSI is neither helpful nor feasible (De Riggi, Moumne, Heath, \& Lewis, 2017; White Kress, Drouhard, \& Costin, 2006). It is thus imperative that everyone involved in the research be aware of the criteria for breaking confidentiality. Coupled with this may be the need for psychoeducation regarding NSSI for school staff.

Ideally, the school administration should designate one or more school personnel (ideally a mental health professional) as contact(s) who will be in the school at all times while the study is underway (see Hasking et al., 2016). The contact(s) should be informed in advance about the nature of the study, the procedure of the notification (best done with the student) and provided with information and materials on best practice in responding to NSSI. A 'confidentiality break' report, signed by both the researcher and the school contact, documents the reason for the confidentiality break, the procedure followed, and indicates that the school contact person is now taking responsibility to follow-up as needed. Finally, there should be no sharing of information with other school personnel (e.g., teachers, administrators) until the designated person has conducted a more detailed evaluation of the situation, as they would with any other referral of potential student risk.

\section{The risk of iatrogenic effects}


Hasking, Conducting research on nonsuicidal self-injury in schools: Ethical considerations and recommendations, 'School Psychology International' (40, 3) pp. 217-234. Copyright @ 2019. DOI: 10.1177/0143034319827056.

There is concern that asking about NSSI, even within a research context, can increase risk of self-injurious thoughts and behaviour (i.e., produce iatrogenic effects). Adolescents are particularly susceptible to peer influence, meaning that risk of these iatrogenic effects may be increased (Albert, Chein, \& Steinberg, 2013). Nevertheless, while discussion or reflection on sensitive topics can be upsetting for some people (Jorm et al., 2007), there is growing evidence that asking about NSSI does not increase distress or the risk of engaging in self-injury. In early work, delivery of a prevention program specifically targeting NSSI in schools produced no adverse effects (Muehlenkamp, Walsh, \& McDade, 2010). In a randomized controlled trial, the presence of a question about NSSI pro- duced no iatrogenic effects (Muehlenkamp, Swenson, Batehan, \& Jarvi, 2015). In another study, students who self-injured were more likely to report being worried or upset by a lengthy questionnaire about mental health concerns (including NSSI); however, the majority reported gaining a better understanding of themselves and others (Hasking et al., 2015). Similarly, Whitlock, Pietrusza, and Purrington (2013) noted that few young adults reported a negative effect of participating in NSSI research. Instead, across these studies, students reported altruistic benefits of participation and that their participation alerted them to available help.

\section{How do we minimize risk of iatrogenic effects?}

Despite the lack of robust evidence for large iatrogenic effects, given the gravity of NSSI and its related outcomes, researchers have an ethical obligation to minimize potential risks of participation. Checklists are a common way to assess the nature and extent of NSSI; however, there is concern that providing a list of methods may inadvertently spark the idea to self-injure or offer new methods to people who had not previously considered them. To combat this, researchers can tailor question- naires to individual respondents. For example, a screening 
question (Have you ever self-injured?) can be used to determine whether more detailed followup questions are revealed to participants. This solution, however, demonstrates the complexities involved in weighing ethical priorities. Use of single-item screeners is associated with lower reported rates of NSSI, potentially missing key information about people who self-injure (Swannell et al., 2014). Thus, a single item screen could be used to minimize the risk of iatrogenic effects, but is used at the expense of scientific integrity. Risk of iatrogenic effects can also be minimized with appropriate duty of care procedures.

\section{Duty of care}

The imperative to do no harm becomes stark when a possible outcome of the behaviour in question is suicide. However, risk needs to be balanced against the benefits of anonymity, the practicalities of following up with all participants in large scale studies, and the evidence regarding the utility of suicide risk assessments. How duty of care is addressed will vary depending on the research design, the constructs under investigation, and the sample (LloydRichardson, Lewis, Whitlock, Rodham, \& Schatten, 2015). In all cases, we would advocate the provi- sion of appropriate resources to all participants (not just those who self-injure). This could include alternative coping strategies (e.g., ways to manage urges) and, when relevant, community-based resources (e.g., distress lines, local counselling services). Resources can be local but also more general for situations in which the geographic location of a participant is unknown (e.g., online studies). Resources may focus on general wellbeing or NSSI specifically. They also may be targeted and action-oriented (e.g., encouragement to go to hospital if urgent).

\section{Duty of care in online studies}

Web-based surveys are increasingly common, providing a cost-effective, convenient means of recruiting data from a large number of students. Moreover, many youth may prefer to 
Hasking, Conducting research on nonsuicidal self-injury in schools: Ethical considerations and recommendations, 'School Psychology International' (40, 3) pp. 217-234. Copyright @ 2019. DOI: 10.1177/0143034319827056.

complete studies via the Internet, given its salience among people who self-injure (Lewis \& Seko, 2016). Another factor to consider is that partici- pants may be more forthcoming in online versus offline research (Barak \& Gluck- Ofri, 2007). Naturally, when participants are not in direct contact with the investigator(s), concerns may arise about the impact of taking part in the research process.

To maximize access to appropriate resources when completing online questionnaires, researchers may wish to ensure that all pages within study websites provide access to these resources (e.g., via hyperlink, textbox). Researchers can also devise their online studies such that respondents receive an automatic pop-up window with resources, contingent on particular responses to questions in the survey (e.g., if students indicate recent NSSI urges). This approach can also be tailored to facilitate participants' access to more active resources (e.g., e-mental health programs; Melvin et al., 2018). The provision of resources based on how individuals respond to particular questions may be especially germane for anonymous online research, when it is not possible to follow-up with participants. Another strategy people can use in online studies includes making use of 'skip logic' and 'display logic' to ensure participants are not unduly exposed to unnecessary details about NSSI. This minimizes risk of iatrogenic effects as well as reduces the risk that participants will become distressed when completing the surveys.

The above strategies notwithstanding, it is incumbent on researchers to ensure that any approach involving the provision of resources (or alternate means to mitigate risk) considers the cultural context of the intended participants. In non- western cultures, reliance on resources developed with a western understanding of NSSI may not be relevant. Thus, attention to how NSSI may be differentially viewed and understood seems warranted. In such cases, resources would ideally be germane to the unique concerns of the targeted group (e.g., translating documents into relevant 
languages).

Finally, distraction tasks or mood augmentation tasks also merit consideration as a means of mitigating psychological upset that may stem from participation in online NSSI research. For example, some researchers have used distraction buttons on study webpages, which participants use on their own accord. By virtue of clicking on the button, individuals are brought to an external website (e.g., Wikipedia, a crossword). From there, they can return to the study when they feel ready (Lloyd-Richardson et al., 2015). Similarly, some researchers use brief videos (e.g., of nature) or relaxation exercises (e.g., progressive muscle relaxation) at the end of the study (e.g., Lewis, Seko, \& Joshi, 2018).

\section{Duty of care in interview studies}

Although one-on-one interviews can allow assessment of imminent risk, research- ers must be mindful that they cannot ethically act in the role of researcher while also providing counselling to participants. For studies involving direct involvement with participants, any limits on confidentiality should not only be included in information sheets but also reviewed orally so that participants can ask questions to ensure they fully understand these limits. It is also essential that interviewers are suitably qualified in interviewing, risk assessment, and managing participant distress. Researchers may also wish to engage participants in stress-reduction techniques or a mood induction as a part of the debriefing process. However, inasmuch as sufficient qualifications are key when conducting interview studies, the same applies for guiding participants through any post-study exercise.

\section{Duty of care in experimental studies}

Some NSSI research involves individuals taking part in experimental tasks that may, themselves, raise ethical concerns. For example, mood induction techniques are sometimes used 
Hasking, Conducting research on nonsuicidal self-injury in schools: Ethical considerations and recommendations, 'School Psychology International' (40, 3) pp. 217-234. Copyright @ 2019. DOI: 10.1177/0143034319827056.

to ascertain how participants experience upset or frustration (e.g., Arbuthnott, Lewis, \& Bailey, 2013; Groschwitz, Plener, Groen, Bonenberger, \& Abler, 2016). Others have used painful tasks to assess differences in pain thresh- olds among adolescents who self-injure and those who did not (Koenig et al., 2017). On the one hand, these lines of inquiry help to obtain a fuller understanding of important NSSI processes. On the other hand, and understandably, these approaches raise concerns about the impact of such tasks on participants.

Clearly, much like other forms of NSSI research, ensuring participants have access to resources is key in the context of experimental studies. However, it is also critical that researchers consider ways to reduce stress and perhaps even aug- ment participants' mood after study completion. Another complexity in using mood manipulation tasks is that they often require the use of deception, in which participants are misled about the task's true purpose. As part of the debrief- ing process, students are advised not to 'spill the beans' to fellow classmates, as this would limit the validity of the task for future participants. However, an unintended consequence of this approach is that researchers are inadvertently advising students not to seek support from their peers if they are still upset by the task. In such cases, it is particularly important that students are no longer upset or distressed when leaving the testing room. We recommend checking if the participant has indeed returned to baseline levels of emotion following any experimental task (and not simply assuming they have).

\section{Engaging schools in research}

Engaging school boards, principals, and other decision-makers in research necessitates that researchers be willing to meet principals, boards of trustees, parent teacher associations, and/or school leadership groups - particularly where self-injury or other sensitive topics may be the focus of research and thus may be seen as potentially signalling a 'problem' to the 
community. Supportive principals, senior leadership, and mental health staff are essential for successful collaboration.

School decision-makers may be hesitant to become involved in self-injury research due to concerns about reputational risk. That is, their community might infer that the school's involvement in such a study means the school 'has a prob- lem' regarding self-injury. The additional workload and resource burden for schools who participate in self-injury research also warrants careful consideration. Schools are busy places and do reasonably decline research involvement on these grounds. In many cases, research with school communities is part of an ongoing collaborative relationship (e.g., Groschwitz et al., 2016).

\section{How do we engage schools in research?}

Approaches to mitigate reputational risk are two-pronged. First, researchers should actively maintain the confidentiality of schools that do (and do not) take part in the research.

Second, researchers should work alongside school leadership and mental health staff to frame the research in a broader context than NSSI. This framing will, to some extent, depend on the nature of the research being conducted, although in general it may be helpful to emphasize to school staff, parents, and students that the research will provide greater understanding of the coping strategies that young people use, and how to best support young people.

Prior to school recruitment, researchers should clearly communicate the workload associated with participation in the research and, where possible, aim to make participation as easy as possible for schools and their staff. Additionally, if research involves protocols for risk assessment and referral of at-risk young people to school mental health staff, these staff should anticipate the workload associated with triage and crisis management and, if necessary, receive resources or financial support from researchers in doing so. Researchers should also work to 
Hasking, Conducting research on nonsuicidal self-injury in schools: Ethical considerations and recommendations, 'School Psychology International' (40, 3) pp. 217-234. Copyright @ 2019. DOI: 10.1177/0143034319827056.

mitigate the burden associated with student participation. In many cases, school-based research occurs during class time. Therefore, researchers should work with school staff to ensure that disruption to education is minimized.

Of course, mental health professionals working in schools can also actively seek opportunities to work with experienced researchers. In doing so, schools may have access to research funding, research assistants, resources, and expertise that can facilitate opportunities for schools to conduct research. Such a partnership also has the benefit of providing school-based researchers with access to appropriate ethical review boards (i.e., in addition to Board of Education approval), who are trained in the principles guiding ethical conduct of research. In line with beneficence principles, researchers should be mindful of current events as they relate to schools. Suicides and other tragedies affect school communities deeply, and the appearance of insensitivity can damage the potential for any future relationship with schools, students, and parents. Beyond minimizing harm, distributive justice principles require that schools and students benefit from engaging in research. In our experience, school mental health professionals and senior leadership find (de-identified) targeted feedback about pertinent issues to be helpful for understanding students' needs and informing resource provisions. Similarly, community presentations about the research are typically well-received. Further, offering professional development opportunities aligned with the researchers' expertise and the schools' needs can be one way to ensure mutual benefits for multiple stakeholders.

Safety for the researcher

An important, but often overlooked, point to consider when conducting school- based self-injury research is the duty of care to the researcher (Bloor, Fincham, \& Sampson, 2007; Moncur, 2013). Unfortunately, this ethical blind spot sits along- side growing evidence that 
conducting research on sensitive topics, such as self- injury, has the potential to create distress, emotional burnout, and feelings of guilt and vulnerability for researchers (Boden, Gibson, Owen, $\&$ Benson, 2016). Risk of psychological or emotional harm may be greater for researchers who seek to cap- ture individual participants' experiences through qualitative or interview-based methodologies (Boden et al., 2016) or who work in isolation (Mckenzie, Li, Jenkin, \& Collings, 2017). Within a school context, the potential for harm to the researcher may be amplified in instances in which the boundaries between the researcher's work and community overlap (Elmird, Schmied, Jackson, \& Wilkes, 2011), as may be the case for researchers who have school-aged children or siblings or who are recruiting participants from their former schools. Establishing regular supervision, debriefing, opportunities for reflection for the research team (including 'peripheral researchers' such as transcribers or experimental confederates) and peermentorship is critical for maintaining researcher safety (for discussion, see Kendall \& Halliday, 2014).

\section{Cultural considerations}

Although we have deliberately focused on broad ethical principles that should underpin all school-based research, there are some cultural considerations that merit attention. Although there is some consistency in prevalence, primary methods, and associations with suicidal thoughts and behaviours, there is variation in the function NSSI serves and inconsistencies in gender patterns across countries (Gholamrezaei et al., 2017; Hanania, Heath, Emery, Toste, \& Daoud, 2015). For example, researchers working in Hong Kong (You, Leung, \& Fu, 2012), India (Kharsati \& Bhola, 2014), and Indonesia (Tresno, Ito, \& Mearns, 2012) have reported more interpersonal reasons for NSSI among their samples (e.g., regulating a social situation), which calls into question the universality of NSSI as a means of affect regulation. Furthermore, gender 
Hasking, Conducting research on nonsuicidal self-injury in schools: Ethical considerations and recommendations, 'School Psychology International' (40, 3) pp. 217-234. Copyright @ 2019. DOI: 10.1177/0143034319827056.

differences in prevalence and method have been found in some contexts (Gholamrezaei et al., 2017). In Jordan, Hanania and colleagues (2015) reported higher prevalence of NSSI in adolescent males than females. Additionally, in a recent meta-analysis of gender patterns in China (Yang \& Feldman, 2018), a complex pattern of gender differences by age and location (urban, rural, Hong Kong/mainland) emerged. Cross-cultural variations in the function of NSSI could have ethical implications; where NSSI is maintained by interpersonal reinforcers, additional considerations around peer influence in the onset and maintenance of NSSI are warranted. Researchers can follow the same processes used to minimize iatrogenic effects talking about NSSI as one form of coping, limiting detailed discussion of methods, and ensuring students have access to appropriate mental health resources.

Differences have also been reported in North America as a function of ethnicity and religious affiliation, although findings are inconsistent. Some researchers have found higher rates of NSSI among Caucasian youth as compared with non- Caucasian, Arab-American and AfricanAmerican youth (Muehlenkamp \& Gutierrez, 2007; Kuentzel, Arble, Boutros, Chugani, \& Barnett, 2012), while others report similar rates among Caucasian, Asian, and other minority students (Whitlock \& Knox, 2007; Whitlock et al., 2011). Religiosity and/or spirituality may be a pro- tective factor (Kress, Newgent, Whitlock, \& Mease, 2015). Christian, Muslim, and Hindu youth have all reported lower rates of NSSI than youth who do not endorse a religion (Borrill, Fox, \& Roger, 2011; Kuentzel et al., 2012); however, whether these discrepancies are a function of religiosity or cultural influences remains untested. Cross-cultural work in this area is in its infancy, and further inquiry is needed to fully understand variations in function, method and gender patterns of NSSI globally. Finally, researchers should not assume that traditional definitions of NSSI make sense across cultures. For example, among Ma- ori (the indigenous 
people of New Zealand), kiri haehae is a common private cultural practice involving cuts to the body and face as an expression of intense grief (Kingi et al., 2017). Additionally, for Ma- ori, the wha- nau (family) is conceived more broadly than is common among pa- keha- (European New Zealanders). In this population, older siblings, or even adults who may not share a genetic relationship with a young person, may occupy caregiver roles and thus expect to be involved in consultation and/or research in a manner that is consistent with these roles.

\section{Conclusion}

Ethics committees typically prioritize beneficence over justice (Omerov, Steineck, Dyregrov, Runeson, \& Nyberg, 2014), yet we urge ethics committees and schools to also consider the benefits that research participation can have. Following the principle of justice, all potential participants deserve to experience the myriad benefits of research, whether those be

feeling an altruistic sense of helping further scientific knowledge or the more tangible benefits of receiving mental health service referrals. We would also argue that it is unethical to not do anything to better understand NSSI; the onus is on researchers, ethics committees, and schools to work together to facilitate this type of research. Only then can we ensure our knowledge of NSSI moves forward and that prevention initiatives, treatments, and policy are scientifically informed. 
Hasking, Conducting research on nonsuicidal self-injury in schools: Ethical considerations and recommendations, 'School Psychology International' (40, 3) pp. 217-234. Copyright @ 2019. DOI: 10.1177/0143034319827056.

\section{References}

Albert, D., Chein, J., \& Steinberg, L. (2013). The teenage brain: Peer influences on adoles- cent decision making. Current Directions in Psychological Science, 22, 114-120. doi: $10.1177 / 0963721412471347$

Arbuthnott, A. E., \& Lewis, S. P. (2015). Parents of youth who self-injure: A review of the literature and implications for mental health professionals. Child and Adolescent Psychiatry and Mental Health, 9, 35. doi:10.1186/s13034-015-0066-3

Arbuthnott, A. E., Lewis, S. P., \& Bailey, H. N. (2013). Rumination and emotions in nonsuicidal self-injury and eating disorder behaviours: A preliminary test of the emo- tional cascade model. Journal of Clinical Psychology, 71, 62-71. doi:10.1002/jclp.22115

Baetens, I., Andrews, T., Claes, L., \& Martin, G. (2015). The association between family functioning and NSSI in adolescence: The mediating role of depressive symptoms. Family Science, 6, 330-337.

Barak, A., \& Gluck-Ofri, O. (2007). Degree and reciprocity of self-disclosure in online forums. Cyberpsychology and Behavior, 10, 407-417.

Bentley, K. H., Cassiello-Robbins, C. F., Vittorio, L., Sauer-Zavala, S., \& Barlow, D. H. (2015). The association between nonsuicidal self-injury and the emotional disorders: A metaanalytic review. Clinical Psychology Review, 37, 72-88. doi:10.1016/j.cpr. 2015.02.006

Bloor, M., Fincham, B. and Sampson, H. (2007). Qualiti (NCRM) Commissioned Inquiry into the risk to well-being of researchers in Qualitative research, Cardiff University.

Boden, Z. V. R., Gibson, S., Owen, G. J., \& Benson, O. (2016). Feelings and intersubject- ivity in qualitative suicide research. Qualitative Health Research, 26, 1078-1090. doi:10.1177/1049732315576709 
Borrill, J., Fox, P., \& Roger, D. (2011). Religion, ethnicity, coping skill and reported self- harm in a diverse non-clinical UK population. Mental Health, Religion, and Culture, 14, 259269. doi:10.1080/13674670903485629

Bracken-Roche, D., Bell, E., Macdonald, M. E., \& Racine, E. (2017). The concept of 'vulnerability’ in research ethics: An in-depth analysis of policies and guidelines. Health Research Policy and Systems, 15, 8. doi:10.1186/s12961-016-0164-6

Canadian Institutes of Health Research, Natural Sciences and Engineering Research Council of Canada, and Social Sciences and Humanities Research Council of Canada, Tri-Council Policy Statement: Ethical Conduct for Research Involving Humans, December 2014.

Council for International Organisations of Medical Sciences (2002). International ethical guidelines for biomedical research involving human subjects. Prepared by the Council for International Organizations of Medical Sciences (CIOMS) in collaboration with the World Health Organization (WHO). Geneva.

De Riggi, M. E., Moumne, S., Heath, N. L., \& Lewis, S. P. (2017). Nonsuicidal self-injury in our schools: A review and research-informed guidelines for school mental health professionals. Canadian Journal of School Psychology, 32, 122-143. doi:10.1177/ 0829573516645563

Elmird, R., Schmied, V., Jackson, D., \& Wilkes, L. (2011). Interviewing people about potentially sensitive topics. Nurse Researcher, 19, 12-16. doi: $10.7748 / \mathrm{nr} 2011.10 .19 .1 .12 . c 8766$

European Union Agency for Fundamental Rights. (2014). Child participation in research. Retrieved from http://fra.europa.eu/en/theme/rights-child/child-participation-in-research Gholamrezaei, M., de Stefano, J., \& Heath, N. L. (2017). Nonsuicidal self-injury across cultures 
Hasking, Conducting research on nonsuicidal self-injury in schools: Ethical considerations and recommendations, 'School Psychology International' (40, 3) pp. 217-234. Copyright @ 2019. DOI: 10.1177/0143034319827056.

and ethnic and racial minorities: A review. International Journal of Psychology, 52, 316-326. doi:10.1002/ijop.12230

Groschwitz, R. C., Plener, P. L., Groen, G., Bonenberger, M., \& Abler, B. (2016). Differential neural processing of social exclusion in adolescents with non-suicidal self- injury: An fMRI study. Psychiatry Research: Neuroimaging, 255, 43-48. doi:10.1016/ j.pscychresns.2016.08.001

Hanania, J. W., Heath, N. L., Emery, A. A., Toste, J. R., \& Daoud, F. A. (2015). Non- suicidal self-injury among adolescents in Amman, Jordan. Archives of Suicide Research, 19, 260-274. doi:10.1080/13811118.2014.915778

Hasking, P., Heath, N. L., Kaess, M., Lewis, S. P., Plener, P. L., Walsh, B. W., Whitlock, J., \& Wilson, M. S. (2016). Position paper for guiding response to non-suicidal self-injury in schools. School Psychology International, 37, 644-663. doi:10.1177/0143034316678656

Hasking, P., Rees, C., Martin, G., \& Quigley, J. (2015). What happens when you tell some- one you self-injure? The effects of disclosing NSSI to adults and peers. BMC Public Health, 15, 1039. doi:10.1186/s12889-015-2383-0

Hasking, P., Tatnell, R., \& Martin, G. (2015). Adolescents' reactions to participating in ethically sensitive research: A prospective self-report study. Child and Adolescent Psychiatry and Mental Health, 9, 39. doi:10.1186/s13034-015-0074-3

Hesson, K., Bakal, D., \& Dobson, K. S. (1993). Professional issues - legal and ethical issues concerning children's rights of consent. Canadian Psychology, 34, 317-328.

International Society for the Study of Self-Injury. (ISSS; 2018). Definition of non-suicidal selfinjury. Retrieved from http://www.itriples.org/isss-aboutself-i.html

Jorm, A. F., Kelly, C. M., \& Morgan, A. J. (2007). Participant distress in psychiatric research: A 
systematic review. Psychological Medicine, 37, 917-926. doi:org/10.1017/

S0033291706009779

Kelada, L., Whitlock, J., Hasking, P., \& Melvin, G. (2016). Parents' experiences of non- suicidal self-injury among adolescents and young adults. Journal of Child and Family Studies, 25(11), 3403-3416. doi:10.1007/s10826-016-0496-4

Kelly, A. B., \& Halford, W. K. (2007). Responses to ethical challenges in conducting research with Australian adolescents. Australian Journal of Psychology, 59, 24-33. doi:10.1080/00049530600944358

Kendell, S., \& Halliday, L. E. (2014). Undertaking ethical qualitative research in public health: Are current ethical processes sufficient?. Australian and New Zealand Journal of Public Health, 38, 306-310.

Kharsati, N., \& Bhola, P. (2014). Patterns of non-suicidal self-injurious behaviours among college students in India. International Journal of Social Psychiatry, 61, 39-49. doi:10.1177/0020764014535755

Kingi, T., Russell, L., Ashby, W., Garisch, J. A., O’Connell, A., \& Wilson, M. S. (2017). Ma- te ma- tau, ka ora: The use of traditional Indigenous knowledge to support contemporary rangatahi Ma- ori who self-injure. New Zealand Journal of Psychology, $46,137-145$.

Koenig, J., Rinnewitz, L., Warth, M., Hillecke, T. K., Brunner, R., Resch, F., \& Kaess, M. (2017). Psychobiological response to pain in female adolescents with nonsuicidal selfinjury. Journal of Psychiatry Neuroscience, 42, 189-199. doi:10.1503/jpn.160074

Kress, V., Newgent, R., Whitlock, J., \& Mease, L. (2015). Spirituality, life satisfaction, and life meaning: Protective factors for non-suicidal self-injury. Journal of College Counselling, 
Hasking, Conducting research on nonsuicidal self-injury in schools: Ethical considerations and recommendations, 'School Psychology International' (40, 3) pp. 217-234. Copyright @ 2019. DOI: 10.1177/0143034319827056.

18, 160-174. doi:10.1002/jocc.12012

Kuentzel, J. G., Arble, E., Boutros, N., Chugani, D., \& Barnett, D. (2012). Nonsuicidal selfinjury in an ethnically diverse college sample. American Journal of Orthopsychiatry, 82, 291-297. doi:10.1111/j.1939-0025.2012.01167.x

Larcher, V., \& Hutchinson, A. (2009). How should paediatricians assess Gillick competence? Archives of Disease in Childhood, 95, 307-311. doi:10.1136/adc.2008.148676

Lewis, S. P., \& Seko, Y. (2016). A double-edged sword: A review of benefits and risks of online nonsuicidal self-injury activities. Journal of Clinical Psychology, 72, 249-262. doi:10.1002/jclp.22242

Lewis, S. P., Seko, Y., \& Joshi, P. (2018). The impact of YouTube peer feedback on attitudes toward recovery from non-suicidal self-injury: An experimental pilot study. Digital Health, 4, 1-7. doi:10.1177/2055207618780499

Lloyd-Richardson, E. E., Lewis, S. P., Whitlock, J. L., Rodham, K., \& Schatten, H. T. (2015). Research with adolescents who engage in non-suicidal self-injury: Ethical considerations and challenges. Child and Adolescent Psychiatry and Mental Health, 9, 37. doi:10.1136/adc.2008.148676

McKenzie, S. K., Li, C., Jenkin, G., \& Collings, S. (2017). Ethical considerations in sensitive suicide research reliant on non-clinical researchers. Research Ethics, 13, 173-183.

Melvin, G. A., Gresham, D., Beaton, S., Coles, J., Tonge, B., Gordon, M., \& Stanley, B. (2018). Evaluating the feasibility and effectiveness of an Australian safety planning smartphone application: A pilot study within a tertiary mental health service. Suicide and LifeThreatening Behavior. Advance online publication. doi:10.1111/sltb.12490 Moncur W. (2013). The emotional well-being of researchers: Considerations for practice. In: 
Proceedings of the SIGCHI Conference on Human Factors in Computing Systems 2013

Muehlenkamp, J. J., \& Gutierrez, P. M. (2007). Risk for suicide attempts among adolescents who engage in non-suicidal self-injury. Archives of Suicide Research, 11, 69-82. doi:10.1080/13811110600992902

Muehlenkamp, J. J., Swenson, L. P., Batejan, K. L., \& Jarvi, S. M. (2015). Emotional and behavioural effects of participating in an online study of nonsuicidal self-injury: An experi- mental analysis. Clinical Psychological Science, 3, 26-37. doi:10.1177/2167702614531579

Muehlenkamp, J. J., Walsh, B. W., \& McDade, M. (2010). Preventing non-suicidal self- injury in adolescents: The signs of self-injury program. Journal of Youth and Adolescence, 39(3), 306-314. doi:10.1007/s10964-009-9450-8

National Commission for the Protection of Human Subjects of Biomedical and Behavioral Research. (1979). The Belmont Report. Ethical principles and guidelines for the protection of human subjects of research. Washington: Department of Health, Education, and Welfare.

National Ethics Advisory Committee. (2012). Ethical Guidelines for Intervention Studies: Revised edition. Wellington: Ministry of Health.

National Health and Medical Research Council (NHMRC). (2007). National Statement on Ethical Conduct in Human Research. Canberra: Australian Government.

Oakland, T., Goldman, S., \& Bischoff, H. (1997). Code of ethics of the International School Psychology Association. School Psychology International, 18, 291-298.

Omerov, P., Steineck, G., Dyregrov, K., Runeson, B., \& Nyberg, U. (2014). The ethics of doing nothing. Suicide-bereavement and research: Ethical and methodological consider- ations. 
Hasking, Conducting research on nonsuicidal self-injury in schools: Ethical considerations and recommendations, 'School Psychology International' (40, 3) pp. 217-234. Copyright @ 2019. DOI: 10.1177/0143034319827056.

Psychological Medicine, 44, 3409-3420. doi:10.1017/S0033291713001670

Ribeiro, J. D., Franklin, J. C., Fox, K. R., Bentley, K. H., Kleiman, E. M., Chang, B. P., \& Nock, M. K. (2016). Self-injurious thoughts and behaviors as risk factors for future suicide ideation, attempts, and death: A meta-analysis of longitudinal studies. Psychological Medicine, 46, 225-236.

Swannell, S., Martin, G., Page, A., Hasking, P., \& St John, N. (2014). Prevalence of nonsuicidal self-injury (NSSI) in nonclinical samples; systematic review, meta-analysis and meta-regression. Suicide and Life-Threatening Behavior, 44, 273-303. doi:10.1111/ sltb. 12070

The Nuremberg Code. (1949). British Medical Journal, 313, 1448. doi:10.1136/ bmj.313.7070.1448

Tresno, F., Ito, Y., \& Mearns, J. (2012). Self-injurious behavior and suicide attempts among Indonesian college students. Death Studies, 36, 627-639.

UK Framework for Health and Social Care Research. (2018). London: Health Research Authority.

United Nations Office of the High Commission. (1990). Convention on the Rights of the Child. Geneva: United Nations.

US Title 45 Code of Federal Regulations, Part 46 (Common Rule; 2009). Department of Health and Human Services.

White Kress, V. E., Drouhard, N., \& Costin, A. (2006). Students who self-injure: School counselor ethical and legal considerations. Professional School Counseling, 10, 203-209. Whitlock, J. L., \& Knox, K. L. (2007). The relationship between self-injurious behavior and suicide in a young adult population. Archives of Pediatrics and Adolescent Medicine, 
$161,634-640$.

Whitlock, J. L., Muehlenkamp, J., Purington, A., Eckenrode, J., Barreira, J., .. . Abrams, G. B. (2011). Non-suicidal self-injury in a college population: General trends and sex differences. Journal of American College Health, 59, 691-698. doi:10.1080/07448481. 2010.529626

Whitlock, J., Pietrusza, C., \& Purrington, A. (2013). Young adult respondent experiences of disclosing self-injury, suicide-related behavior, and psychological distress in a web-based survey. Archives of Suicide Research, 17, 20-32.

World Medical Association. (1964). The Declaration of Helsinki: Ethical principles for medical research involving human subjects. Helsinki, Finland: 18th World Medical Assembly.

Yang, X., \& Feldman, M.W. (2018). A reversed gender pattern? A meta-analysis of gender differences in the prevalence of non-suicidal self-injurious behaviour among Chinese adolescents. BMC Public Health, 18, 66.

You, J., Leung, F., \& Fu, K. (2012). Exploring the reciprocal relations between nonsuicidal selfinjury, negative emotions and relationship problems in Chinese adolescents: A longitudinal cross-lag study. Journal of Abnormal Child Psychology, 40, 829-836. doi:10.1007/s10802-011-9597-0 
Hasking, Conducting research on nonsuicidal self-injury in schools: Ethical considerations and recommendations, 'School Psychology International' (40, 3) pp. 217-234. Copyright @ 2019. DOI: 10.1177/0143034319827056.

\section{Tables and Figures}

Table 1. Recommended strategies to ensure ethical conduct of NSSI research. 
Respect for persons

Beneficence

Justice
- Provide details of:

- Aims of the project

- Participation requirements

- Risk and benefits of participation

- How privacy will be protected

- How participants can access results

- Who to contact with questions

- Consider whether parental consent is always required

- Consider competency of students to provide their own consent

- Provide explicit examples of questions that will be asked, so potential participants can decide if they wish to reflect on the content

- Be clear about withdrawal of participation and/or data

- Be clear about limits of confidentiality

- Be mindful of recent events in schools that may render schools vulnerable in the context of the research

- Where possible, arrange for a school-based mental health professional to be onsite both during and after the study

- Provide links to mental health resources for all participants

- Avoid explicit descriptions of NSSI methods

- Provide distraction screens in online studies

- In online studies, program diversions to appropriate resources if participants indicate distress or urges to self-injure

- Use skip logic in online questionnaires to ensure unnecessary detail is not presented to all participants

- Use a mood induction task to return participants to a calm and relaxed state after the study is finished

- Ensure researchers are competent to conduct the study

- Ensure researchers are competent to conduct any required risk assessment

- Reconsider conceptualization of youth or people who self-injure as vulnerable groups, and consider their vulnerability in the context of the specific research project

- Allow everyone who is interested and capable to be in the study

- Work with schools and ethics committees to find solutions to ethical dilemmas

- Engage the whole school community in the research process 